\title{
Skeletal Muscle Index at Intensive Care Unit Admission Is a Predictor of Intensive Care Unit-Acquired Weakness in Patients With Sepsis
}

\author{
Yuta Mitobe $^{\mathrm{a}}$, Shinichiro Morishita ${ }^{\mathrm{b}}$, Kazuki Ohashic ${ }^{\mathrm{c}}$, Sayuri Sakai ${ }^{\mathrm{d}}$, Mieko Uchiyama ${ }^{\mathrm{d}}$, \\ Hansani Abeywickrama ${ }^{\mathrm{d}}$, Etsuko Yamada ${ }^{\mathrm{d}}$, Yuko Kikuchi ${ }^{\mathrm{d}}$, Masakazu Nitta ${ }^{\mathrm{e}}$, \\ Tadayuki Honda ${ }^{\text {f }}$, Hiroshi Endoh ${ }^{f}$, Shinji Kimurag, Shuhei Sakanog, Yu Koyamad, h
}

\begin{abstract}
Background: Intensive care unit-acquired weakness (ICU-AW) can be diagnosed using the Medical Research Council (MRC) score. However, such scoring may not be possible in ICU patients who may be sedated or delirious or have encephalopathy. Currently, a quantitative assessment of the cross-sectional area of the muscle is available to assess changes in skeletal muscle mass using computed tomography (CT) images. This assessment calculates the skeletal muscle index (SMI) $\left(\mathrm{cm}^{2} / \mathrm{m}^{2}\right)$ by dividing the cross-sectional area $\left(\mathrm{cm}^{2}\right)$ of the skeletal muscle at the level of the third lumbar vertebra by the square of the patient's height $\left(\mathrm{m}^{2}\right)$ on CT. This study assessed the effectiveness of SMI, as measured by abdominal CT scans, in predicting the onset of ICU-AW in patients with sepsis admitted to the ICU.
\end{abstract}

Methods: We examined septic ICU patients admitted to the Niigata University Hospital ICU during 2012 - 2017 under mechanical ventilation. Patients were retrospectively divided into two groups by MRC score at ICU discharge: group AW comprised patients with an MRC score $<48$, and group non-AW (NAW) comprised the remaining pa-

Manuscript submitted October 19, 2019, accepted November 11, 2019

aDepartment of Nursing, Faculty of Medical Technology, Teikyo University, 2-11-1 Kaga, Itabashi, Tokyo 173-8605, Japan

bDepartment of Physical Therapy, Niigata University of Health and Welfare, Shimami-cho 1398, Kita-ku, Niigata 950-3198, Japan

${ }^{\circ}$ Department of Nursing, Sapporo University of Health Science, 2-1-15, Nakanumanishi 4-jo, Sapporo Shi Higashi Ku, Hokkaido 007-0894, Japan

dDepartment of Nursing, Niigata University Graduate School of Health Sciences, 2-746 Asahimachi, Niigata, Niigata 951-8518, Japan

${ }^{\mathrm{e} A d v a n c e d ~ E m e r g e n c y ~ a n d ~ C r i t i c a l ~ C a r e ~ C e n t e r, ~ N i i g a t a ~ U n i v e r s i t y ~ M e d i c a l ~}$ and Dental Hospital, 1-754 Asahimachi, Niigata, Niigata 951-8520, Japan

fDivision of Emergency and Critical Care Medicine, Niigata University Graduate School of Medical and Dental Sciences, 1-757 Asahimachi, Niigata, Niigata 951-8510, Japan

gRehabilitation Center, Niigata University Medical and Dental Hospital, 1-754 Asahimachi, Niigata, Niigata 951-8520, Japan

${ }^{\mathrm{h}}$ Corresponding Author: Yu Koyama, Department of Nursing, Niigata University Graduate School of Health Sciences, 2-746 Asahimachi, Niigata, Niigata 951-8518, Japan. Email: yukmy@clg.niigata-u.ac.jp

doi: https://doi.org/10.14740/jocmr4027 tients. Clinicopathological factors at ICU admission such as age, gender, underlying disease, body mass index, and SMI were compared between the two groups. Statistical analyses were performed using the Mann-Whitney U test, Fisher's exact test, receiver operator characteristic (ROC) analysis and multivariate analysis.

Results: A total of 31 septic patients were examined, and 23 patients met the criteria for ICU-AW. The prevalence of women was significantly higher in group AW $(\mathrm{P}<0.05)$. All clinical factors, except for gender, were not significantly different between the two groups. SMI was significantly lower in group AW than in group NAW (P $<0.05)$. ROC analysis revealed that the cut-off value of SMI for predicting ICU-AW was 44.1, and the multivariate analysis revealed that only low SMI was a significant factor in predicting ICU-AW (P $<0.05)$.

Conclusions: Our results show that SMI measurement at ICU admission is a valid predictive factor for ICU-AW progression in septic patients.

Keywords: Intensive care unit-acquired weakness; Skeletal muscle index; Computed tomography; Sepsis

\section{Introduction}

Intensive care unit-acquired weakness (ICU-AW) is a complication in severely ill patients who require mechanical ventilation (MV), and it results in systemic muscle weakness $[1,2]$. Approximately $60-100 \%$ of patients with sepsis have ICU-AW $[3,4]$. In the short term, ICU-AW can cause delayed ventilator weaning, prolonged ICU stays, and prolonged hospitalization $[5,6]$. In the long term, ICU-AW limits the activities of daily living after discharge and leads to a reduction in the quality of life $[7,8]$.

ICU-AW can be diagnosed using the Medical Research Council (MRC) score; a total MRC score of $<48$ on the 60-point assessment meets the criteria for a diagnosis of ICUAW $[9,10]$. However, performing muscular tests requires cooperation and consciousness [11], and this may not be possible in ICU patients as they may be sedated or delirious or have 
encephalopathy [12]. Therefore, the diagnosis of ICU-AW in ICU patients lags a few days behind the actual onset of the condition [13]. However, timely diagnosis of ICU-AW will reduce the short- and long-term outcomes of ICU-AW even in ICU patients on sedatives or with encephalopathy or delirium. Currently, a quantitative assessment of the cross-sectional muscle area using computed tomography (CT) images is available to assess changes in skeletal muscle mass [14]. This assessment calculates the skeletal muscle index (SMI) $\left(\mathrm{cm}^{2} /\right.$ $\mathrm{m}^{2}$ ) by dividing the cross-sectional area $\left(\mathrm{cm}^{2}\right)$ of the skeletal muscle at the level of the third lumbar vertebra (L3) by the square of the patient's height $\left(\mathrm{m}^{2}\right)$ on CT [15]. SMI measured using CT may reflect MRC scores. SMI may be used to diagnose ICU-AW.

Because patients with sepsis require timely and appropriate therapeutic interventions for infection foci, CT imaging upon ICU admission is usually performed to identify such foci $[16,17]$. Therefore, the SMI obtained from CT imaging can be used to identify patients at risk of developing ICU-AW and prevent its progression. The present study assessed the effectiveness of SMI, as measured by abdominal CT scans, in predicting the onset of ICU-AW in patients with sepsis admitted to the ICU.

\section{Materials and Methods}

\section{Study design, setting, population, and approval to conduct this study}

We retrospectively examined patients treated for sepsis at the Emergency Critical Care Center, Niigata University Medical and Dental Hospital, between April 2012 and March 2017. Ethical approval to conduct this study was granted by the Niigata University Ethics Committee (approval ID: 20170135). Consent was obtained from all patients before data were acquired from their medical charts. We used opt-out manner in the present study. The disclosure document about this clinical study were informed through the website of Niigata University Graduate School of Health Sciences and also posted on Niigata University Medical and Dental Hospital's bulletin board. This study was conducted in accordance with the Declaration of Helsinki. Severe sepsis and septic shock were defined according to the guidelines published by the Surviving Sepsis Campaign in 2012 [16]. Patients were eligible for inclusion if they 1) were admitted to the ICU due to sepsis; 2) were on a mechanical ventilator; 3) had an abdominal CT scan at both ICU admission and discharge; and 4) had an MRC score measured at ICU discharge. We excluded patients who 1 ) were $\geq 80$ years or $<19$ years of age; 2) required assistance or had gait disturbances before hospitalization; 3) did not have an MRC score measured; 4) were admitted to the ICU twice or more during the study period; 5) had congenital or progressive nervous/muscular diseases; or 6) died during hospitalization. Our reason for excluding patients with ages of $\geq 80$ years was that patients who develop septic disorders at such late ages are regarded as having poor prognoses [18].

\section{Data collection}

\section{Medical record review}

We extracted data from the medical charts to obtain the demographic and clinical information of the patients on ICU admission such as height, body weight, body mass index (BMI), Barthel index, Acute Physiology and Chronic Health Evaluation (APACHE) II score, basic disorders, length of ICU and hospital stay, days until first upright sitting, days until first sitting in a wheelchair, blood purification therapy, MV days, and CT images.

\section{Laboratory results}

Blood data were obtained from each patient at the time of both ICU admission and ICU discharge for biochemical and hematological evaluation and included test results for serum albumin (Alb), blood urea nitrogen (BUN), creatinine (Cre), C-reactive protein (CRP), aspartate aminotransferase (AST), alanine aminotransferase (ALT), total bilirubin (T-bil), creatine kinase $(\mathrm{CK})$, and white blood cell count (WBC).

\section{Imaging analysis}

The cross-sectional area of the skeletal muscle at the level of L3 was measured on a single axial image using semi-automated, threshold-based segmentation (thresholds -29 and +150 Hounsfield units) [19] in ImageJ (version 1.51, National Institutes of Health, Bethesda, Maryland) [20]. The L3 slice provides information on a number of muscles: the erector spinae, quadratus lumborum, psoas, transversus abdominis, interior and exterior obliques, and rectus abdominis. The software computed a muscle surface area in $\mathrm{cm}^{2}$ by multiplying the pixel area by the number of pixels identified as muscle.

\section{Diagnosis of ICU-AW}

The MRC score was evaluated using a 0 - 5 manual muscle strength test for six joint movements including upper-extremity shoulder abduction, elbow flexion, wrist extension, lowerextremity hip flexion, knee extension, and ankle flexion. An MRC score below 48 is one of the criteria for diagnosing ICUAW [9]. According to the results of the MRC score assessment, patients were classified into two groups: group AW and group non-AW (NAW).

\section{Statistical analysis}

Statistical analyses were performed using the Mann-Whitney U test and Fisher's exact test to compare group AW and group NAW at ICU admission and discharge. Multivariate logistic regression analysis was used to identify patient factors predic- 


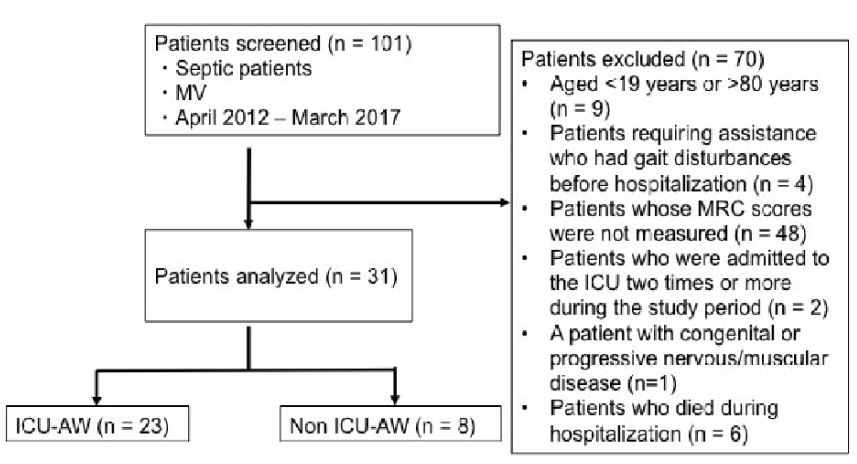

Figure 1. Study flowchart. MV: mechanical ventilator; ICU-AW: intensive care unit-acquired weakness; MRC: Medical Research Council.

tive of ICU-AW diagnosis. A P value of $<0.05$ was considered significant. Receiver operator characteristic (ROC) analysis was used to identify the appropriate SMI cut-off values that predicted ICU-AW. We split into groups lower SMI and higher SMI based on SMI cut-off value. Wieske et al reported candidate predictors for ICU-AW [21]. Five factors were relevant in our study (lactate, glucose, $\mathrm{pH}$, age and gender). Multivariate logistic regression analysis was used to identify patient factors predictive of ICU-AW diagnosis. Statistical analysis was performed using SPSS (version 24, IBM Corp., Armonk, NY, USA).

\section{Results}

\section{Patient characteristics at baseline}

A total of 101 patients were enrolled, and 23 patients $(74 \%)$ were diagnosed with ICU-AW. We excluded nine patients who were $>80$ years or $<19$ years of age, four patients who required assistance or had gait disturbances before hospitalization, 48 patients whose MRC score was not measured, two patients who were admitted to the ICU twice or more during the study period, one patient with a congenital or progressive nervous/muscular disease, and six patients who died during hospitalization. Ultimately, the remaining 31 patients were included in the analyses (Fig. 1).

\section{Patient background}

Of the 31 enrolled patients, 23 patients were classified in group AW, and eight patients were classified in group NAW. The average age of the group AW was 66.0 years (standard deviation (SD): 11.9 years; range: 31 - 75 years). Group NAW was 66.1 years (SD: 13.3 years; range: 37 - 76 years). Figure 2 shows the prevalence of various underlying diseases in group AW and group NAW. In both groups, the most common sepsis etiology was pneumonia. At baseline, there was a significantly lower proportion of men in group AW than in group NAW $30 \% \mathrm{vs}$. $75 \%, \mathrm{P}=0.043)$. There were no significant differences in other baseline characteristics between the two groups (Table 1).

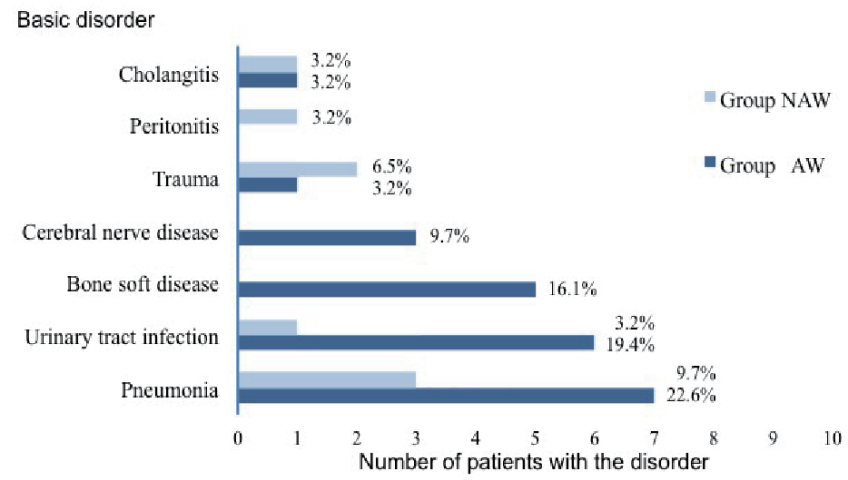

Figure 2. Prevalence of various underlying basic disorders in both groups of patients with sepsis.

\section{Comparison of laboratory parameters}

There were no significant differences in Alb, BUN, Cre, CRP, glucose, AST, ALT, T-bil, CK, WBC, partial pressure of oxygen $\left(\mathrm{PO}_{2}\right), \mathrm{pH}$, and partial pressure of carbon dioxide $\left(\mathrm{PCO}_{2}\right)$ between the two groups at ICU admission or at ICU discharge (Table 2).

\section{Factors associated with ICU discharge and hospital dis- charge}

The BMI for both groups (AW and NAW) was comparable ( 23.3 vs. $\left.22.8 \mathrm{~kg} / \mathrm{m}^{2}, \mathrm{P}=0.874\right)$. There was no significant difference in MV days between the two groups. Patients in group AW had significantly longer hospital lengths of stay (LOSs) (95.5 days vs. 32.3 days, $\mathrm{P}=0.007)$, more days until the first upright sitting (25.4 days vs. 8.8 days, $\mathrm{P}=0.015)$, more days until first sitting in a wheelchair (26.2 days vs. 9.2 days, $\mathrm{P}=$ 0.007 ), and higher Barthel index values (49.7 vs. 81.2, $\mathrm{P}=$ 0.021 ) than patients in group NAW (Table 3 ).

\section{SMI assessments}

Days from admission to CT imaging and from ICU discharge to CT were comparable between the two groups. SMI at ICU admission was significantly lower in group AW than in group NAW $\left(40.7 \mathrm{~cm}^{2} / \mathrm{m}^{2}\right.$ vs. $\left.46.4 \mathrm{~cm}^{2} / \mathrm{m}^{2}, \mathrm{P}=0.042\right)$. SMI at ICU discharge was significantly lower in group AW than in group NAW (36.9 $\mathrm{cm}^{2} / \mathrm{m}^{2}$ vs. $\left.43.4 \mathrm{~cm}^{2} / \mathrm{m}^{2}, \mathrm{P}=0.029\right)$ (Table 4).

\section{Factors predictive of ICU-AW}

Based on a univariate analysis, the following variables were selected as independent variables for the logistic regression analysis of the incidence of ICU-AW: gender, length of hospital stay, days until first upright sitting, days until first sitting in a wheelchair, Barthel index at hospital discharge, SMI at ICU admission, and SMI at ICU discharge. Logistic regression analy- 
Table 1. Comparison of Baseline Characteristics Between Groups With and Without ICU-AW

\begin{tabular}{llll} 
& Group AW $(\mathbf{n = 2 3 )}$ & Group NAW $(\mathbf{n}=\mathbf{8})$ & P value \\
\hline Age (years) & $66.0 \pm 11.9$ & $66.1 \pm 13.3$ & 0.511 \\
Gender, male (\%) & $7(30.4 \%)$ & $6(75.0 \%)$ & 0.043 \\
BMI $\left(\mathrm{kg} / \mathrm{m}^{2}\right)$ & $24.5 \pm 5.0$ & $24.3 \pm 1.9$ & $<0.999$ \\
Height & $155.7 \pm 11.9$ & $162.0 \pm 13.1$ & 0.182 \\
Weight (kg) & $63.1 \pm 14.7$ & $59.6 \pm 14.2$ & 0.443 \\
Barthel index & $13.0 \pm 19.6$ & $16.2 \pm 16.6$ & 0.305 \\
APACHE II score & $22.8 \pm 8.8$ & $17.8 \pm 4.1$ & 0.167 \\
\hline
\end{tabular}

Data are expressed as means \pm standard deviation. Categorical variables were compared using Fisher's exact test, and continuous variables were compared using the Mann-Whitney U test. BMI: body mass index; APACHE: Acute Physiology and Chronic Health Evaluation; ICU-AW: AW: acquired weakness; NAW: non-acquired weakness.

Table 2. Comparison of Laboratory Parameters Between Groups With and Without ICU-AW at ICU Admission and ICU Discharge

\begin{tabular}{|c|c|c|c|c|c|c|}
\hline & \multicolumn{3}{|c|}{ ICU admission } & \multicolumn{3}{|c|}{ ICU discharge } \\
\hline & Group AW $(n=23)$ & Group NAW $(n=8)$ & P value & Group AW $(n=23)$ & Group NAW $(n=8)$ & P value \\
\hline $\mathrm{Alb}(\mathrm{g} / \mathrm{dL})$ & $2.08 \pm 0.4$ & $1.9 \pm 0.4$ & 0.496 & $2.1 \pm 0.4$ & $2.1 \pm 0.3$ & 0.602 \\
\hline Cre (mg/dL) & $1.9 \pm 1.3$ & $2.9 \pm 2.7$ & 0.892 & $1.1 \pm 1.4$ & $0.6 \pm 0.3$ & 0.391 \\
\hline CRP (mg/dL) & $24.5 \pm 9.6$ & $24.1 \pm 6.8$ & 0.964 & $8.7 \pm 7.9$ & $8.7 \pm 7.9$ & 0.964 \\
\hline Glucose (mg/dL) & $155.3 \pm 108.9$ & $142.5 \pm 57.3$ & 0.701 & $121.2 \pm 28.7$ & $136 \pm 30.2$ & 0.13 \\
\hline AST (U/L) & $349.1 \pm 925.9$ & $132.7 \pm 185.2$ & 0.857 & $31.3 \pm 21.3$ & $42.7 \pm 24$ & 0.175 \\
\hline ALT(U/L) & $248.2 \pm 763.8$ & $60.5 \pm 71.1$ & 0.786 & $33.6 \pm 35.3$ & $52.7 \pm 38.5$ & 0.114 \\
\hline T-bil (mg/dL) & $1.54 \pm 1.2$ & $1.8 \pm 1.1$ & 0.442 & $0.9 \pm 1$ & $1 \pm 0.7$ & 0.275 \\
\hline CK (U/L) & $887.3 \pm 1435.6$ & $1117.1 \pm 1733.6$ & 0.685 & $47.3 \pm 80.1$ & $101 \pm 134.1$ & 0.175 \\
\hline WBC $\left(10^{3} / \mu \mathrm{L}\right)$ & $9536.5 \pm 7131.5$ & $8405.0 \pm 6932.1$ & 0.498 & $9536.5 \pm 7131.5$ & $8405 \pm 6932.1$ & 0.391 \\
\hline
\end{tabular}

Data are expressed as means \pm standard deviation. Categorical variables were compared using Fisher's exact test, and continuous variables were compared using the Mann-Whitney U test. Alb: serum albumin; BUN: blood urea nitrogen; Cre: creatinine; CRP: C-reactive protein; AST: aspartate aminotransferase; ALT: alanine aminotransferase; T-bil: total bilirubin; CK: creatine kinase; WBC: white blood cell count; ICU-AW: intensive care unitacquired weakness; AW: acquired weakness; NAW: non-acquired weakness.

Table 3. Factors Associated With ICU Discharge and Hospital Discharge

\begin{tabular}{llll} 
& Group AW (n= 23) & Group NAW (n=8) & P value \\
\hline BMI & $23.3 \pm 5.4$ & $22.8 \pm 2.1$ & 0.874 \\
ICU LOS (day) & $23.4 \pm 20.6$ & $11.8 \pm 8.3$ & 0.214 \\
Hospital LOS (day) & $95.5 \pm 61.9$ & $32.3 \pm 14.1$ & 0.007 \\
Days until first upright sitting (day) & $25.4 \pm 35.6$ & $8.8 \pm 8$ & 0.015 \\
Days until first sitting in a wheelchair (day) & $26.2 \pm 27.2$ & $9.2 \pm 7.9$ & 0.007 \\
Blood purification therapy (\%) & $7(30.4 \%)$ & $1(12.5 \%)$ & 0.642 \\
Barthel index & $49.7 \pm 33.3$ & $81.2 \pm 21.1$ & 0.021 \\
MV day & $5.9 \pm 7.5$ & $2.6 \pm 2.2$ & 0.243 \\
\hline
\end{tabular}

Data are expressed as means \pm standard deviation or as $n(\%)$. Categorical variables were compared using Fisher's exact test, and continuous variables were compared using the Mann-Whitney U test. BMI: body mass index; ICU LOS: intensive care unit length of stay; MV: mechanical ventilation; AW: acquired weakness; NAW: non-acquired weakness. 
Table 4. Assessment of Skeletal Muscle Index

\begin{tabular}{|c|c|c|c|c|c|c|}
\hline & \multicolumn{3}{|c|}{ ICU admission } & \multicolumn{3}{|c|}{ ICU discharge } \\
\hline & Group AW $(n=23)$ & Group NAW $(n=8)$ & $P$ value & Group AW $(n=23)$ & Group NAW $(n=8)$ & P value \\
\hline Days until CT imaging & $0.5 \pm 1.3$ & $1.3 \pm 2.5$ & 0.241 & $18.1 \pm 11.8$ & $14.3 \pm 5.7$ & 0.825 \\
\hline
\end{tabular}

Data are expressed as means \pm standard deviation. Categorical variables were compared using Fisher's exact test, and continuous variables were compared using the Mann-Whitney U test. CT: computed tomography; SMI: skeletal muscle index; ICU: intensive care unit; AW: acquired weakness; NAW: non-acquired weakness.

sis showed that only SMI at ICU admission was an independent predictive factor for developing ICU-AW (odds ratio (OR): $1.417 ; 95 \%$ confidence interval $(\mathrm{CI}): 1.024-1.960 ; \mathrm{P}=0.035)$.

\section{Comparing SMI at ICU admission with ICU-AW diagnosis}

Because SMI at ICU admission was the only predictor of ICUAW, we performed an ROC analysis to determine the cut-off value of SMI at ICU admission. Figure 3 shows the predictive ability of SMI at ICU admission in identifying ICU-AW (cutoff value of SMI at ICU admission: $44.1 \mathrm{~cm}^{2} / \mathrm{m}^{2} ; \mathrm{P}=0.042$ ). The relationship between ICU-AW and the cut-off value for SMI at ICU admission is shown in Table 5. Patients with SMI $>44.1 \mathrm{~cm}^{2} / \mathrm{m}^{2}$ were less likely to have ICU-AW than those with $\mathrm{SMI} \leq 44.1 \mathrm{~cm}^{2} / \mathrm{m}^{2}(16.1 \%$ vs. $58.0 \%, \mathrm{P}=0.012)$. The Logistic regression analysis explanatory variables used lactate, glucose, $\mathrm{pH}$, age, gender and ICU-AW as explanatory variables. Patients with $\mathrm{SMI} \leq 44.1 \mathrm{~cm}^{2} / \mathrm{m}^{2}$ were approximately twice as likely to have ICU-AW as patients with $\mathrm{SMI}>44.1$

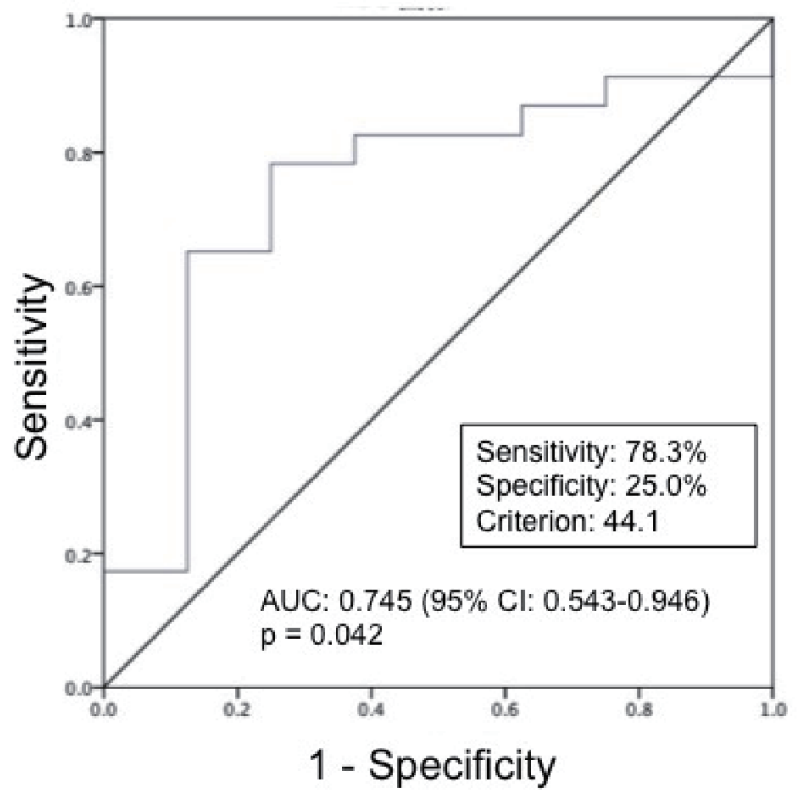

Figure 3. ROC curves for SMI at ICU admission in prediction of ICUAW $(n=31)$. ROC: receiver operating characteristic; SMI: skeletal muscle index; ICU-AW: intensive care unit-acquired weakness. The ROC analysis identified a cut-off point of $44.1 \mathrm{~cm}^{2} / \mathrm{m}^{2}$ (area under the curve: 0.745 ; sensitivity $78.3 \%$; specificity $25.0 \%$; $P=0.042$ ). $\mathrm{cm}^{2} / \mathrm{m}^{2}$ (OR: 10.800; 95\% CI: 1.644 - 70.934; $\left.\mathrm{P}=0.013\right)(\mathrm{Ta}-$ ble 6).

\section{Discussion}

\section{Prevalence of ICU-AW}

This study showed that the prevalence of ICU-AW in patients with sepsis was $74.1 \%$, which is within the $60-100 \%$ range reported in previous studies $[3,4]$. Diagnosis of ICU-AW is based on the MRC score, which is difficult to assess in patients with an altered state of consciousness due to sedation or brain pathology [12]. In 2017, Harvey et al reported that ICU-AW occurs in $25-80 \%$ of patients who are on MV for at least 4 days and in $50-75 \%$ of patients with sepsis [22]. Similarly, in our study, the mean duration of MV for patients with ICU-AW was 5.9 days, and the prevalence of ICU-AW in patients with sepsis was $74.1 \%$.

\section{Comparison with previous studies}

In this study, the sensitivity and specificity for SMI at ICU admission were $78.3 \%$ and $25.0 \%$, respectively. In a previous study that used early electrophysiological testing in patients admitted to the ICU, the sensitivity and specificity of direct muscle stimulation after 3 days of ICU admission were $83 \%$ and $89 \%$, respectively [23]. This indicates that direct muscle stimulation may have a better discriminative performance than SMI. However, direct muscle stimulation is invasive and technically challenging to perform, and therefore, it is not used widely.

In another study, ICU-AW was predicted based on factors such as age, treatment with aminoglycosides, and highest lactate levels during the first 2 days of ICU admission [21]. We found that SMI could be used to predict ICU-AW at an earlier time point than that mentioned in previous studies.

\section{Relationship between ICU-AW and SMI}

There were no significant differences in ICU LOS, hospital LOS, or MV days between the two groups. However, group AW showed longer ICU LOSs, longer hospital LOSs, and more MV days than group NAW. In the short term, ICU-AW delays ventilator weaning [5] and prolongs the length of ICU 
Table 5. Comparison of Skeletal Muscle Index With ICU-AW

\begin{tabular}{llll}
\hline & Lower SMI $(\mathbf{n}=\mathbf{2 0})$ & Higher SMI $(\mathbf{n}=\mathbf{1 1})$ & Pvalue \\
\hline ICU-AW + & $18(58.0)$ & $5(16.1)$ & 0.012 \\
ICU-AW - & $2(6.4)$ & $6(19.3)$ & \\
\hline
\end{tabular}

Data are expressed as $\mathrm{n}(\%) . \mathrm{P}<0.05$ was considered significant. Categorical variables were compared by Fisher's exact test. ICU-AW: intensive care unit-acquired weakness; SMI: skeletal muscle index.

stay and hospitalization [6].

We found that group AW had a significantly smaller SMI at ICU admission than group NAW. Muscle wasting in acute illness reportedly becomes clinically apparent at $2-4$ weeks from illness onset. However, in the present study, ICU-AW patients had their SMI evaluated on average only 0.5 days after admission. The early identification of skeletal muscle loss in our study is thought to have been due to several factors. For example, patients with sepsis in the ICU might have had muscle loss due to an infection before ICU admission. Khan et al [24] reported the prevalence and time of onset of neuromuscular dysfunction in patients with severe sepsis. The majority of patients demonstrated changes in nerve conduction studies within 7 days after ICU admission in the course of severe sepsis. In that study, the changes observed in the nerve conduction studies were predictive of acquired neuromuscular dysfunction development in ICU patients. Axer et al [25] demonstrated that sepsis patients have impairment of sensory nerve fibers in the first week of ICU stay. In the study by Kubiak et al [26], the morphometric analysis was performed on a CT scan obtained on average only 5.7 days after ICU admission, and sepsis patients had muscle atrophy of the greater psoas muscle.

Early skeletal muscle loss at the time of ICU admission may occur earlier in time planning than reported regarding morphometric analysis and neuromuscular pathology. The current study showed that a low SMI at ICU admission was predictive of ICU-AW. According to a study by Moisey et al [27] published in 2013, the in-hospital mortality rate was approximately two-fold higher in patients with a low SMI at ICU admission (sarcopenia) than in those with a high SMI (32\% vs. $14 \%$ ). Moreover, in a retrospective study performed in elderly patients admitted to the ICU, psoas muscle mass at ICU admission was related to ICU LOS and patient outcomes [27]. In another retrospective study, CT images obtained within 4 days of admission to the ICU showed that low skeletal muscle area was a risk factor for death in seriously ill patients on MV, regardless of sex and APACHE score [28]. Patients with low skeletal muscle mass at ICU admission have extended hospital LOS and MV, which is similar to that observed in the case of ICU-AW. Based on the findings of the present study, SMI at
ICU admission can be considered to be a predictor of ICU-AW. Thus, SMI at ICU admission is likely to be a useful metric in future ICU-AW prediction studies. Our study was the first to assess the SMI of ICU-AW patients.

\section{Limitations}

Because there are some limitations in the present study, the results should be interpreted with caution. Firstly, the difference in gender distribution may affect the result of significantly lower SMI in group AW than in group NAW. In a study by Prado et al [29] SMI at the L3 level measured using CT images was $52.4 \mathrm{~cm}^{2} / \mathrm{m}^{2}$ for men and $38.5 \mathrm{~cm}^{2} / \mathrm{m}^{2}$ for women. Although there were differences in the gender distribution between the two groups, gender was not an independent predictor of ICUAW onset in the multivariate analysis. Therefore, we did not set separate SMI values for men and women. However, further studies are necessary to determine whether cut-off values should differ by gender. Secondly, the study information was collected retrospectively. While objective findings were readily available for analysis (such as vital signs and laboratory values), the detail, clarity, and importance of subjective clinical findings could not be categorized or assessed. Thirdly, this was a single-center study with a limited number of patients. Fourthly, ICU admission and ICU discharge practices vary significantly across centers. It is therefore difficult to generalize our findings in other settings. Fifthly, we did not collect lifestyle data. SMI at ICU admission may be related to lifestyle factors, so future studies should clarify the relationship between lifestyle factors and SMI. Lastly, the timing of CT scanning was not standardized which might have caused selection bias. In patients with sepsis, CT scanning was performed just before ICU admission for the purpose of diagnosis. However, CT scans are not conducted for routine monitoring.

\section{Conclusions}

This study aimed to assess the effectiveness of SMI in predict-

Table 6. Logistic Regression Analysis of Variables Associated With Skeletal Muscle Index

\begin{tabular}{llll}
\hline Variable & OR $(\mathbf{9 5 \%} \mathbf{C I})$ & P value & Percentage of correct classifications \\
\hline $\begin{array}{l}\text { SMI at ICU admission } \\
>44.1 \mathrm{~cm}^{2} / \mathrm{m}^{2}\end{array}$ & 1.000 & \\
$\quad \leq 44.1 \mathrm{~cm}^{2} / \mathrm{m}^{2}$ & $10.800(1.644-70.934)$ & 0.013 & $77.4 \%$ \\
\hline
\end{tabular}

Data were analyzed by logistic regression analysis model. OR: odds ratio; Cl: confidence interval; SMI: skeletal muscle index; ICU: intensive care unit. 
ing the onset of ICU-AW in patients with sepsis admitted in the ICU. The SMI at ICU admission was lower in patients with ICU-AW than in those without ICU-AW. Therefore, assessment of SMI at ICU admission may be valuable in the timely diagnosis of ICU-AW. Furthermore, the present results showed that SMI measurements at ICU admission can predict ICUAW progression in septic patients.

\section{Acknowledgments}

We thank Professor Yongbum Lee from the Department of Radiological Technology, Graduate School of Health Sciences, Niigata University, for his helpful advice on the image CT analysis methods.

\section{Financial Disclosure}

This work was supported by JSPS KAKENHI Grant (number: JP1919577).

\section{Conflict of Interest}

The authors declare that there is no conflict of interest.

\section{Informed Consent}

Informed consents were obtained from all patients.

\section{Author Contributions}

Yuta Mitobe, Yu Koyama, Shuhei Sakano and Mieko Uchiyama designed the research; Shuhei Sakano, Yuta Mitobe, Kazuki Ohashi, Hansani Abeywickrama, Etsuko Yamada and Yuko Kikuchi conducted the research; Masakazu Nitta, Tadayuki Honda, Hiroshi Endoh and Shinji Kimura collected the CT data; Yuta Mitobe, Shinichiro Morishita and Sayuri Sakai analyzed the data; Yuta Mitobe summarized the data; Yuta Mitobe and Yu Koyama wrote the manuscript; and Yu Koyama had primary responsibility for the final content. All authors read, checked, and approved the final manuscript.

\section{References}

1. De Jonghe B, Sharshar T, Lefaucheur JP, Authier FJ, Durand-Zaleski I, Boussarsar M, Cerf C, et al. Paresis acquired in the intensive care unit: a prospective multicenter study. JAMA. 2002;288(22):2859-2867.

2. Needham DM, Davidson J, Cohen H, Hopkins RO, Weinert C, Wunsch H, Zawistowski C, et al. Improving longterm outcomes after discharge from intensive care unit: report from a stakeholders' conference. Crit Care Med. 2012;40(2):502-509.
3. Berek K, Margreiter J, Willeit J, Berek A, Schmutzhard E, Mutz NJ. Polyneuropathies in critically ill patients: a prospective evaluation. Intensive Care Med. 1996;22(9):849855.

4. De Jonghe B, Cook D, Sharshar T, Lefaucheur JP, Carlet J, Outin H. Acquired neuromuscular disorders in critically ill patients: a systematic review. Groupe de Reflexion et d'Etude sur les Neuromyopathies En Reanimation. Intensive Care Med. 1998;24(12):1242-1250.

5. De Jonghe B, Bastuji-Garin S, Durand MC, Malissin I, Rodrigues $\mathrm{P}$, Cerf C, Outin $\mathrm{H}$, et al. Respiratory weakness is associated with limb weakness and delayed weaning in critical illness. Crit Care Med. 2007;35(9):2007-2015.

6. Ali NA, O'Brien JM, Jr., Hoffmann SP, Phillips G, Garland A, Finley JC, Almoosa K, et al. Acquired weakness, handgrip strength, and mortality in critically ill patients. Am J Respir Crit Care Med. 2008;178(3):261-268.

7. Herridge MS, Cheung AM, Tansey CM, Matte-Martyn A, Diaz-Granados N, Al-Saidi F, Cooper AB, et al. One-year outcomes in survivors of the acute respiratory distress syndrome. N Engl J Med. 2003;348(8):683-693.

8. Herridge MS, Tansey CM, Matte A, Tomlinson G, DiazGranados N, Cooper A, Guest CB, et al. Functional disability 5 years after acute respiratory distress syndrome. N Engl J Med. 2011;364(14):1293-1304.

9. Stevens RD, Marshall SA, Cornblath DR, Hoke A, Needham DM, de Jonghe B, Ali NA, et al. A framework for diagnosing and classifying intensive care unit-acquired weakness. Crit Care Med. 2009;37(10 Suppl):S299-308.

10. Kleyweg RP, van der Meche FG, Schmitz PI. Interobserver agreement in the assessment of muscle strength and functional abilities in Guillain-Barre syndrome. Muscle Nerve. 1991;14(11):1103-1109.

11. Vanpee G, Hermans G, Segers J, Gosselink R. Assessment of limb muscle strength in critically ill patients: a systematic review. Crit Care Med. 2014;42(3):701-711.

12. Fan E, Zanni JM, Dennison CR, Lepre SJ, Needham DM. Critical illness neuromyopathy and muscle weakness in patients in the intensive care unit. AACN Adv Crit Care. 2009;20(3):243-253.

13. Hough CL, Lieu BK, Caldwell ES. Manual muscle strength testing of critically ill patients: feasibility and interobserver agreement. Crit Care. 2011;15(1):R43.

14. Cruz-Jentoft AJ, Baeyens JP, Bauer JM, Boirie Y, Cederholm T, Landi F, Martin FC, et al. Sarcopenia: European consensus on definition and diagnosis: Report of the European Working Group on Sarcopenia in Older People. Age Ageing. 2010;39(4):412-423.

15. Tsuji M, Amiya E, Hatano M, Nitta D, Maki H, Bujo C, Saito A, et al. Abdominal skeletal muscle mass as a predictor of mortality in Japanese patients undergoing left ventricular assist device implantation. ESC Heart Fail. 2019;6(3):526-535.

16. Dellinger RP, Levy MM, Rhodes A, Annane D, Gerlach H, Opal SM, Sevransky JE, et al. Surviving Sepsis Campaign: international guidelines for management of severe sepsis and septic shock, 2012. Intensive Care Med. 2013;39(2):165-228.

17. De Waele JJ. Early source control in sepsis. Langenbecks 
Arch Surg. 2010;395(5):489-494.

18. Inoue S, Suzuki-Utsunomiya K, Okada Y, Taira T, Iida Y, Miura N, Tsuji T, et al. Reduction of immunocompetent T cells followed by prolonged lymphopenia in severe sepsis in the elderly. Crit Care Med. 2013;41(3):810-819.

19. Mitsiopoulos N, Baumgartner RN, Heymsfield SB, Lyons W, Gallagher D, Ross R. Cadaver validation of skeletal muscle measurement by magnetic resonance imaging and computerized tomography. J Appl Physiol (1985). 1998;85(1):115-122.

20. Fortin M, Battie MC. Quantitative paraspinal muscle measurements: inter-software reliability and agreement using OsiriX and ImageJ. Phys Ther. 2012;92(6):853864.

21. Wieske L, Witteveen E, Verhamme C, Dettling-Ihnenfeldt DS, van der Schaaf M, Schultz MJ, van Schaik IN, et al. Early prediction of intensive care unit-acquired weakness using easily available parameters: a prospective observational study. PLoS One. 2014;9(10):e111259.

22. Harvey MA, Davidson JE. Postintensive care syndrome: right care, right now... and later. Crit Care Med. 2016;44(2):381-385.

23. Weber-Carstens S, Deja M, Koch S, Spranger J, Bubser F, Wernecke KD, Spies CD, et al. Risk factors in critical illness myopathy during the early course of critical illness: a prospective observational study. Crit Care. 2010;14(3):R119.
24. Khan J, Harrison TB, Rich MM, Moss M. Early development of critical illness myopathy and neuropathy in patients with severe sepsis. Neurology. 2006;67(8):14211425.

25. Axer H, Grimm A, Pausch C, Teschner U, Zinke J, Eisenach S, Beck S, et al. The impairment of small nerve fibers in severe sepsis and septic shock. Crit Care. 2016;20:64.

26. Kubiak CA, Ranganathan K, Matusko N, Jacobson JA, Wang SC, Park PK, Levi BL. Computed Tomography Evidence of Psoas Muscle Atrophy Without Concomitant Tendon Wasting in Early Sepsis. J Surg Res. 2019;234:210-216.

27. Moisey LL, Mourtzakis M, Cotton BA, Premji T, Heyland DK, Wade CE, Bulger E, et al. Skeletal muscle predicts ventilator-free days, ICU-free days, and mortality in elderly ICU patients. Crit Care. 2013;17(5):R206.

28. Weijs PJ, Looijaard WG, Dekker IM, Stapel SN, Girbes AR, Oudemans-van Straaten HM, Beishuizen A. Low skeletal muscle area is a risk factor for mortality in mechanically ventilated critically ill patients. Crit Care. 2014;18(2):R12.

29. Prado CM, Lieffers JR, McCargar LJ, Reiman T, Sawyer MB, Martin L, Baracos VE. Prevalence and clinical implications of sarcopenic obesity in patients with solid tumours of the respiratory and gastrointestinal tracts: a population-based study. Lancet Oncol. 2008;9(7):629-635. 